\title{
Template-dependent multiple displacement amplification for profiling human circulating RNA
}

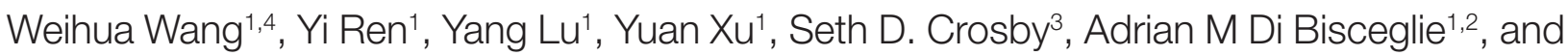 \\ Xiaofeng Fan ${ }^{1,2^{*}}$ \\ ${ }^{1}$ Division of Gastroenterology \& Hepatology, Department of Internal Medicine, Saint Louis University School \\ of Medicine, St. Louis, MO, 2Saint Louis University Liver Center, Saint Louis University School of Medicine, \\ St. Louis, MO, ${ }^{3}$ Department of Genetics, Washington University School of Medicine, St, Louis, MO, and \\ ${ }^{4}$ Wuhan Pulmonary Hospital, Wuhan, Hubei, China
}

\author{
BioTechniques 63:21-27 (July 2017) doi 10.2144/000114566 \\ Keywords: multiple displacement amplification; C18 spacer; liquid biopsy
}

Supplementary material for this article is available at www.BioTechniques.com/article114566.

Multiple displacement amplification (MDA) is widely used in whole-genome/transcriptome amplification. However, template-independent amplification (TIA) in MDA is a commonly observed phenomenon, particularly when using high concentrations of random hexamer primers and extended incubation times. Here, we demonstrate that the use of random pentamer primers with $5^{\prime}$ ends blocked by a C18 spacer results in MDA solely in a template-dependent manner, a technique we have named tdMDA. Together with an optimized procedure for the removal of residual genomic DNA during RNA extraction, tdMDA was used to profile circulating RNA from $0.2 \mathrm{~mL}$ of patient sera. In comparison to regular MDA, tdMDA demonstrated a lack of quantifiable DNA amplification in the negative control, a remarkable reduction of unmapped reads from Illumina sequencing ( $7 \pm$ $10.9 \%$ versus $58.6 \pm 39 \%, P=0.006)$, and increased mapping rates of the serum transcriptome $\left(26.9 \pm 7.9 \%\right.$ versus $\left.5.8 \pm 8.2 \%, P=3.8 \times 10^{-4}\right)$. Transcriptome profiles could be used to separate patients with chronic hepatitis $\mathrm{C}$ virus (HCV) infection from those with HCV-associated hepatocelIular carcinoma (HCC). We conclude that tdMDA should facilitate RNA-based liquid biopsy, as well as other genome studies with biological specimens having ultralow amounts of genetic material.

Multiple displacement amplification (MDA) is a method of isothermal DNA amplification frequently applied to whole-genome amplification (1). MDA typically utilizes phi29 DNA polymerase, which shows exceptional processivity along with strong stranddisplacement and exonuclease activity. As a consequence, MDA outperforms PCR-based approaches in terms of template coverage and amplification fidelity (2). In addition, stranddisplacement amplification results in a hyper-branched product with a high molecular weight ( $\geq 10 \mathrm{~kb})$, which is ideal for library construction in nextgeneration sequencing (NGS). For these reasons, MDA, in combination with NGS, has become a gold standard method in genomic research (3-5). A long-standing issue in MDA is templateindependent amplification (TIA), particularly when amplifying ultralow concentrations of templates that require high concentrations of random hexamer primers and an extended incubation period (1). Positive amplification due to TIA in negative controls not only compromises confidence in experimental results, but, more impor- tantly, generates junk DNA (1). Even when using well-optimized MDA protocols, junk DNA may compose up to $70 \%$ of the product $(1,6,7)$. Elimination of junk DNA has attracted significant investigation, including the use of dimethyl sulfoxide (DMSO) $(8,9)$, T4 gene 32 (9), trehalose (10), single-stranded DNA binding protein (SSB) (11), random hexamer primers containing an internal C3 spacer (12), RNA primer (13), chimeric DNA-RNA primer (14), modulation of incubation temperature (15), and the reduction of reaction volume (16). However, these

\section{METHOD SUMMARY}

Random pentamer primers blocked at the $5^{\prime}$ end with a C18 spacer were shown to eliminate template-independent amplification, a major problem in multiple displacement amplification. 
No Group Name

Sequence $\left(5^{\prime} \rightarrow 3^{\prime}\right)$

MDA yield $(\mu \mathrm{g})$

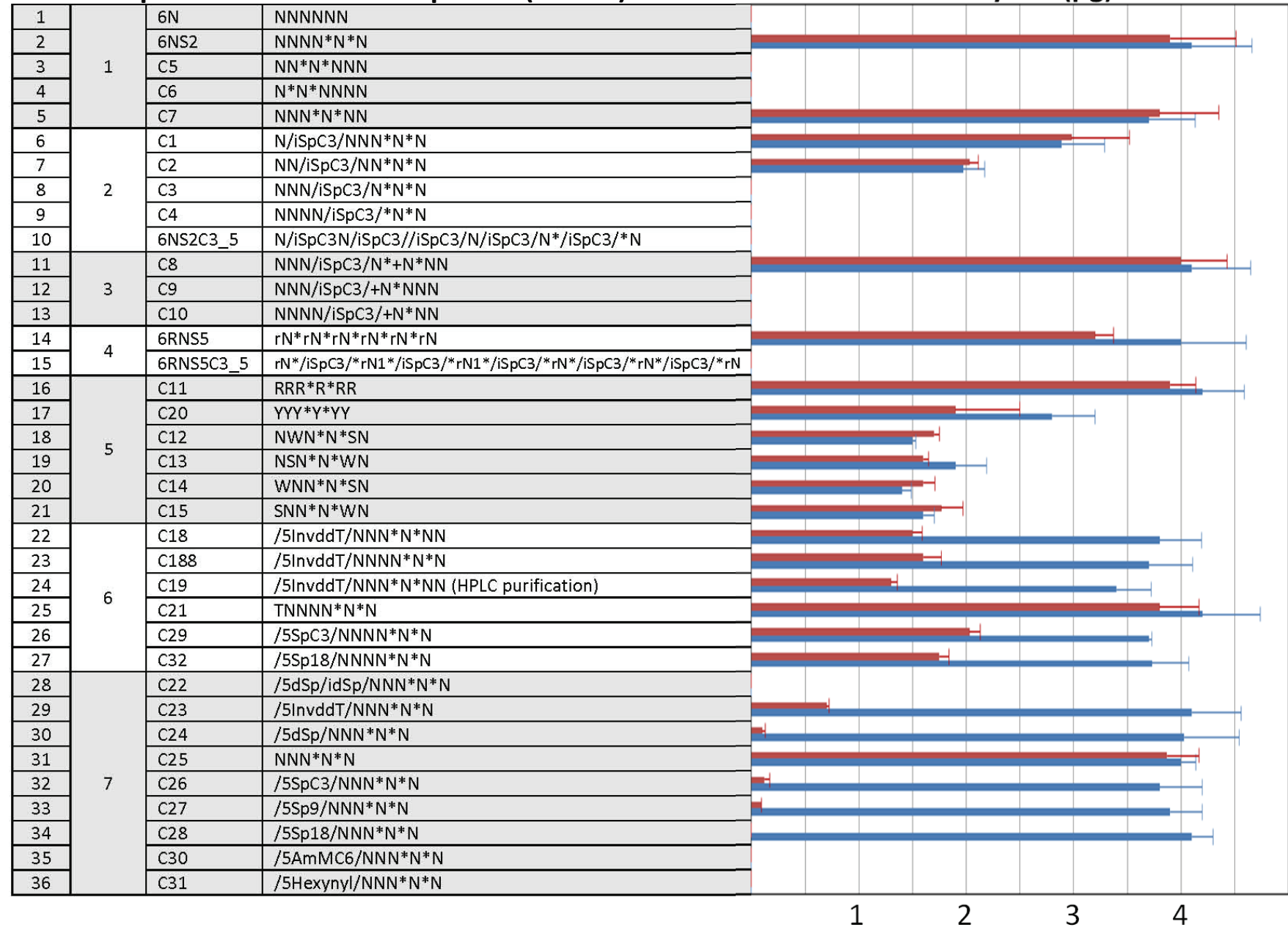

Figure 1. List of primers tested in multiple displacement amplification (MDA). Group 1 primers have phosphorothioate bonds placed at different positions to test the minimum lengths of primers capable of initiating DNA polymerization. Group 2 primers are modified internally with a C3 spacer to minimize self-priming. Group 3 primers are the group 2 primers modified with the inclusion of locked nucleic acid (LNA) to stabilize the binding between primers and templates. Groups 4 and 5 are constrained primers and RNA primers (with or without a C 3 spacer), respectively. Random primers blocked at the $5^{\prime}$ end are designated as group 6 (hexamer) or group 7 (pentamer) primers. In these two groups of primers, the blockage at the $5^{\prime}$ end was tested using different modifications, including inverted, 2',3'dideoxy-dT base (5' inverted ddT), dSpacer, C3 spacer, C9 spacer, C18 spacer, amino modifier C6 (AmMC6) or alkyne. Degenerate bases are indicated using standard International Union of Pure and Applied Chemistry (IUPAC) codes. Each primer was tested in triplicate at a concentration of $50 \mu \mathrm{M}$ in the standard MDA protocol with (blue) or without (red) template [1 ng of the hepatitis C virus (HCV) plasmid]. The MDA reaction with the group 7 primers was incubated at $28^{\circ} \mathrm{C}$, while the other groups of primers were amplified at $30^{\circ} \mathrm{C}$. The error bars indicate SD.

options have not been widely adopted. Increasing the amount of template $(\geq 10$ ng) and using a short incubation time, as recommended in many commercial kits such as the REPLI-g kits from QIAGEN, is a common option to minimize TIA, yet such an amount (10 ng) is not always available in many biological scenarios. Here, we have developed a robust MDA protocol that is free of TIA through the use of $5^{\prime}$ end-blocked random pentamer primers. Isothermal amplification occurs solely in a template-dependent manner, which we have named tdMDA. The potential of tdMDA has been demonstrated by an efficient profiling of human circulating RNA, a biological source notorious for its ultralow concentration and complex heterogeneity (17).

\section{Materials and methods}

Patient samples

A total of 30 serum samples were used in the current study. Of these, 20 were from a completed study (18), and 3 samples from blood donors were used to optimize the experimental protocols for RNA extraction, genomic DNA removal, and tdMDA. The remaining seven samples were collected from patients at the Saint Louis University Hospital. Three patients had chronic HCV infection, and the other four were diagnosed with HCV-associated hepatocellular carcinoma (HCC) by computed tomography (CT), serum $\alpha$ fetoprotein (AFP) measurement, and histological examination. These samples were used to test the feasibility of circulating RNA profiling through the use of tdMDA.

\section{Ethics statement}

For the collection of serum samples, written informed consent was obtained from each patient and was approved by the local Institutional Review Board in the parental studies (19). The research protocol for the collection and use of patient samples was reviewed and approved by the Saint Louis University 
Institutional Review Board (IRB protocol: SLU15565).

\section{Standard MDA protocol}

In order to facilitate experimental optimization, we defined a standard MDA protocol with a reaction volume of $40 \mu \mathrm{L}$ and consisting of phi29 DNA polymerase and its corresponding buffer, $1 \mathrm{mM}$ of dNTPs, and $2 \mu \mathrm{M}$ or $50 \mu \mathrm{M}$ of random exonuclease-resistant primers. A recombinant 12-kb plasmid (HCV plasmid) containing a 9-kb HCV genotype 1a genome from our previous study (20) was used as a positive control. The reaction was incubated at $30^{\circ} \mathrm{C}$ for 16 $\mathrm{h}$, followed by heat-inactivation at $65^{\circ} \mathrm{C}$ for $10 \mathrm{~min}$. In addition to visualization on a $0.8 \%$ agarose gel, MDA yield was quantitated by the Quant-iT PicoGreen dsDNA Assay kit (QIAGEN, Valencia, CA) after purification using the QIAamp DNA mini kit. Due to the extreme sensitivity of the techniques (5), all MDA experiments were performed in strict adherence to the recommendations of Champlot et al. to avoid possible contamination (21).

\section{Elimination of TIA in MDA}

The extreme sensitivity of MDA also causes confusion between TIA and reagent contamination, wherein the latter is caused by trace amounts of bacterial or other microbial genomes left during manufacture. Without additional experimentation, it was difficult to clarify if the positive amplification in negative controls resulted from TIA or reagent contamination. Therefore, a multi-step strategy was applied to eliminate TIA in MDA. First, using the standard MDA protocol, we evaluated the purity of phi29 DNA polymerases from various vendors, including Lucigen (Middleton, WI), Epicentre (Madison, WI), Thermo Scientific (St. Louis, MO), and New England Biolabs (Ipswich, MA). Decontamination through UV irradiation as described by Woyke et al. was also adopted for the reagents, including phi29 DNA polymerase, dNTPs, and primers (8). The procedure was conducted with the Stratagene Stratalinker 2400 UV Crosslinker (Agilent Technologies, Santa Clara, CA). Next, using the standard MDA protocol with reagents that were free of contamination, elimination of TIA was evaluated by the use additives such as DMSO (0.5 5\%)
(Sigma-Aldrich, St. Louis, MO), Escherichia coli SSB (50 300 $\mathrm{gg} / \mathrm{mL})$ (New England Biolabs), and Fc400 (4 64 $\mathrm{mg} / \mathrm{mL}$ ) (Sigma-Aldrich) to emulate a crowded intracellular environment (22). The incubation temperature was tested from $25^{\circ} \mathrm{C}$ to $35^{\circ} \mathrm{C}$.

Finally, we focused on primer optimization by designing a total of 36 different types of primers that could be categorized into 7 groups (Figure 1). Primers containing locked nucleic acid (LNA) were synthesized by Exiqon (Woburn, MA). All remaining primers were purchased from Integrated DNA Technologies (IDT, Coralville, IA). Each primer was tested in triplicate at $50 \mu \mathrm{M}$, a typical concentration used in MDA $(1,2)$.

Serum RNA extraction, genomic DNA removal, and real-time RT-tdMDA Total RNA was extracted from $200 \mu \mathrm{L}$ serum using the miRNeasy Serum/ Plasma kit (QIAGEN) and then eluted into $14 \mu \mathrm{L}$ water. Extracted RNA was quantitated using the Quant-iT RiboGreen RNA kit (Life Technologies,

Micro RNA, small RNA, mRNA, FFPE RNA, Total RNA, ribo-depleted R InRNA, snRNA, Micro RNA, small RNA, mRNA, FFPE RNA, Total RNA, ribo-depleted RNA, InRNA, snRNA, Micro RNA, small RNA, mRNA, FF RNA, Total RNA, ribo-depleted RNA, InRNA, SnRNA, Micro RNA, small mRNA, FFPE RNA, Total RNA, ribo-depleted RNA, InRNA, snRNA, Micr RNA, small RNA, mRNA, FFPE RNA, Total RNA, ribo-depleted RNA, Inf snRNA, Micro RNA, small RNA, mainA FF" ENNA, Total RNA, ribo-der

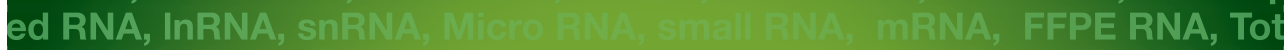
RNA, ribo-depleted RNA InFA AIFIA, small RNA, MRNA

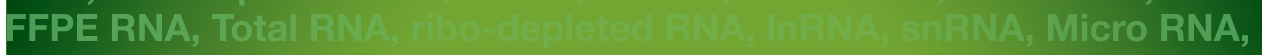

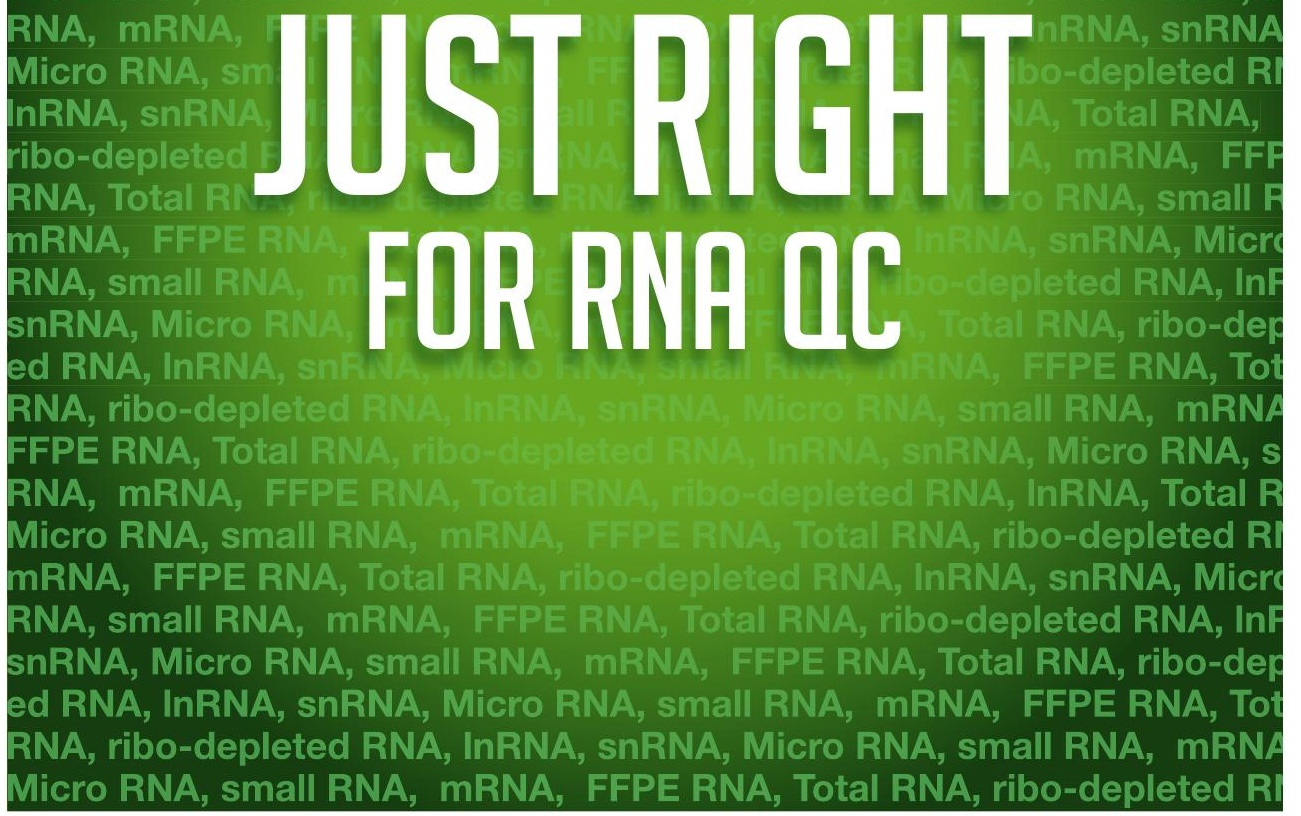

\section{TRANSFORMING RNA ANALYSIS WITH AUTOMATED SAMPLE QC.}

The Fragment Analyzer ${ }^{\mathrm{TM}}$ simultaneously quantifies and qualifies RNA samples, whether you're performing total RNA, mRNA or small RNA analysis, or working with degraded materials like formalin fixed tissues.

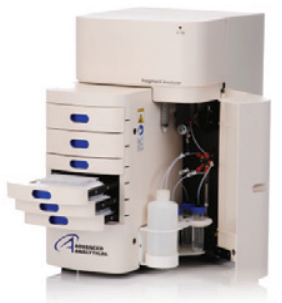




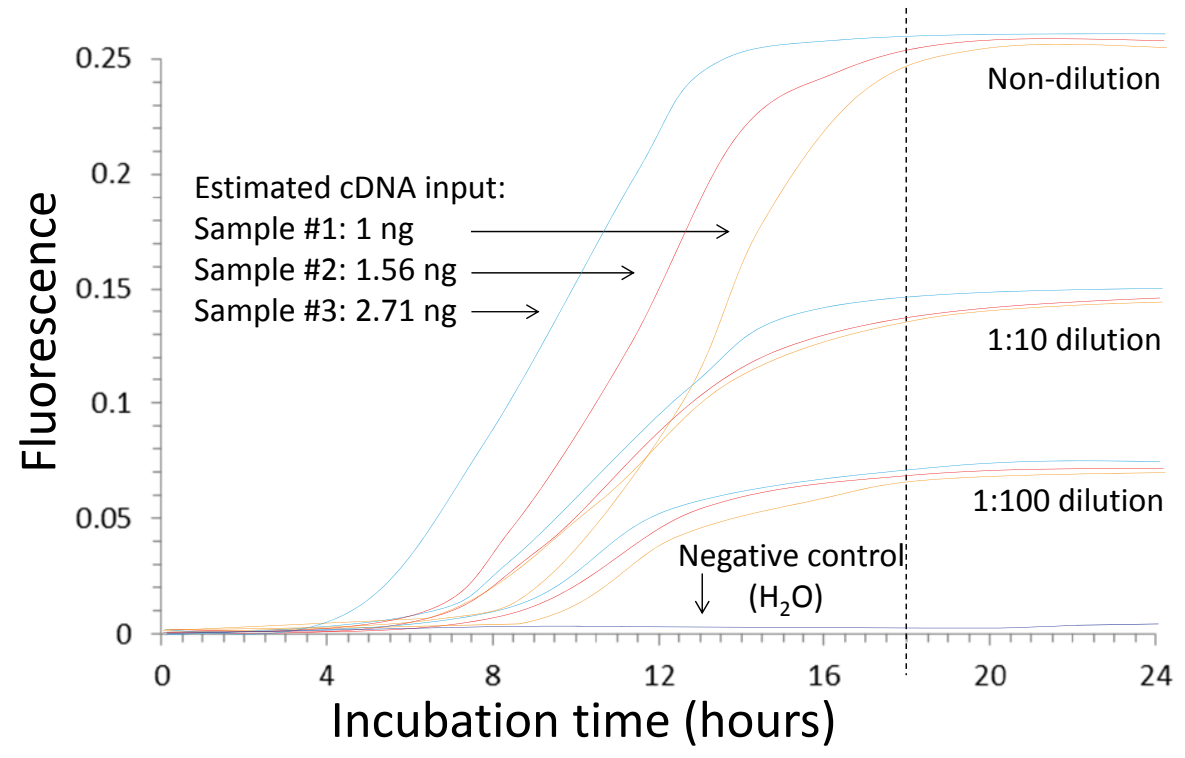

Figure 2. Real-time reverse transcription-template dependent multiple displacement amplification (RT-tdMDA) using three hepatitis C virus (HCV) patient serum samples and a negative control $\left(\mathbf{H}_{2} \mathbf{O}\right)$. These samples covered the range of the RNA yield extracted from $200 \mu \mathrm{L}$ serum (7.5-19.2 $\mathrm{ng}$ ), as quantitated in the final $14 \mu \mathrm{L}$ of elution by the QIAGEN miRNA kit prior to HL-DNase digestion. An aliquot of $10.6 \mu \mathrm{L}$ RNA was used for RT in a reaction containing 200 U SuperScript III, $80 \mu \mathrm{M} 5^{\prime}$-end-blocked random pentamer primer (5'-/iSpC3/NNN*N*N-3'; asterisks denote phosphorothioate bonds), and $2 \mathrm{mM}$ dNTPs in a $20-\mu \mathrm{L}$ volume. An aliquot of $4 \mu \mathrm{L}$ of the RT reaction was used in a 40- $\mu \mathrm{L}$ tdMDA reaction containing $300 \mathrm{U}$ phi29 DNA polymerase (Epicentre), $80 \mu \mathrm{M}$ primer, and 0.1× SYBR Green I (Thermo Fisher Scientific). The reaction was incubated at $28^{\circ} \mathrm{C}$ for $24 \mathrm{~h}$ on the ABI TaqMan 7500 , in which fluorescent intensities were monitored through the SYBR Green channel. Estimated amount of cDNA input in tdMDA $=[10.6 /(14+0.5($ HL-DNase $)+1.4$ (buffer) $] \times[$ total RNA amount $] \times 0.2$. Templateindependent amplification was completely inhibited, as indicated by the negative control.

St. Louis, MO) on a fluorescence microplate reader. Five options were tested to remove residual genomic DNA during RNA extraction (Supplementary Table S1) using a 12-kb HCV plasmid as a spike-in DNA that was added to serum prior to RNA extraction (1 $\mu \mathrm{g} \mathrm{HCV}$ plasmid per $200 \mu \mathrm{L}$ serum). Using purified RNA as the template, a 30-cycle HCV non-structural gene 3 (NS3)-specific PCR was conducted to determine the efficiency of genomic DNA removal.

Purified RNA $(9.4 \mu \mathrm{L})$ was reverse transcribed in a $20 \mu \mathrm{L}$ reaction consisting of $1 \times$ SuperScript III buffer (Life Technologies), $10 \mathrm{mM}$ DTT, 2 mM dNTPs, $20 \cup$ RNasein RNase Inhibitor (Promega, Madison, WI), 200 U SuperScript III reverse transcriptase and $80 \mu \mathrm{M}$ primers. The reaction was incubated at $37^{\circ} \mathrm{C}$ for $30 \mathrm{~min}, 50^{\circ} \mathrm{C}$ for $30 \mathrm{~min}$, and then inactivated at $70^{\circ} \mathrm{C}$ for $15 \mathrm{~min}$. An aliquot of $4 \mu \mathrm{L}$ of this reverse transcription (RT) reaction served as the template in the subsequent MDA. Both RT and MDA used the same random primer capable of elimi- from the Nextera XT DNA Sample Preparation kit (Illumina, San Diego, CA), which added adapter sequences simultaneously. Fragmented DNA was amplified with 12 cycles of PCR using the Index 1 and Index 2 primers from Illumina, followed by purification and size selection using AMPure XP beads (Beckman Coulter, Beverly, MA). Sizeselected libraries were normalized using the normalization beads from Illumina. In this step, the quantity of each library was normalized to ensure equal representation in the pooled sample. An aliquot of $2 \mu \mathrm{L}$ normalized and pooled library was added to 998 $\mu \mathrm{L}$ pre-chilled hybridization buffer, denatured at $98^{\circ} \mathrm{C}$ for $2 \mathrm{~min}$, and then sequenced on the Illumina NextSeq 500 Sequencing platform $(1 \times 250$-bp single reads and mid-output) at MOgene (St. Louis, MO).

\section{Data analysis}

Raw sequence reads in fasta format were first filtered in PRINSEQ (v0.19.5) for quality control, including read length $\geq 70$ bp, mean read quality score $\geq 25$, Iow complexity with DUST score $\leq 7$, ambiguous bases $\leq 1 \%$, and all duplicates (18). By subtracting the human reference genome, NCBI reference sequences for bacteria, viruses and fungi, and NCBI nt database (6), we calculated and compared the rates of unmapped sequencing reads for each sample amplified by either tdMDA or regular MDA. Potential gene detection and differential gene expression (DGE) between HCV and HCC patients were analyzed by the TopHat-featureCountedgeR pipelines $(23,24)$. In brief, filtered reads were mapped in TopHat onto the annotated human reference genome (Ensembl GRCh 38.84). Gene-counting data were summarized at the transcriptlevel from TopHat output in featureCounts (25), followed by DGE analysis in edge $\mathrm{R}$ with the false discovery rate (FDR) set at $10 \%$.

\section{Statistical analysis}

Except for built-in statistical analyses in programs such as Cufflinks and edgeR, other comparisons were done using the two-tailed Student's t-test. Data were expressed as the mean $\pm \mathrm{SD}$, and $P<0.05$ was considered statistically significant. 


\section{Data availability}

Raw sequence data in fasta format from the seven HCV and HCC patient samples was deposited in the NCBI Sequence Read Archive (SRA) under the SRA accession no. SRP071026.

\section{Results and discussion}

Reagent contamination and TIA are two major concerns in MDA. While these issues are negligible when using large amounts of template, both concerns become significant shortcomings when working with low or ultralow amounts of template (typically $<1 \mathrm{ng}$ ) since they compress the product space of target molecules and/or aggravate qualitative biases $(2,6,7,26)$. While reagent contamination could be minimized or even eliminated through the use of clean enzymes and reagents $(27,28)$, TIA remains a consistent issue in MDA, despite many attempts to overcome it. The current study provides a simple, but efficient solution in which the use of a specially designed primer (C28) (Figure 1) eliminates TIA.

In a stepwise optimization process, we first identified that phi29 DNA polymerase from Epicentre was considerably free of reagent contamination in comparison to other enzymes (Supplementary Figure S1). UV treatment of the Epicentre phi29 DNA polymerase eliminated contamination, but MDA yield was reduced, while UV treatment of dNTPS or random hexamer primers abolished or compromised the reaction (Supplementary Figure S2). In the standard MDA protocol using the phi29 enzyme from Epicentre, all additives had no apparent role in eliminating TIA (Supplementary Figure S2). Incubation temperatures $>31^{\circ} \mathrm{C}$ reduced yield, while temperatures $<30^{\circ} \mathrm{C}$ enhanced TIA (Supplementary Figure S2). These results led us to focus on primer design to eliminate TIA in MDA.

As expected from the strong $3^{\prime} \rightarrow 5^{\prime}$ exonuclease activity of phi29 DNA polymerase, which digests oligonucleotides (29), Primer $6 \mathrm{~N}$ of the Group 1 primers produced no DNA amplification. Primers C5 and C6 failed to amplify the HCV plasmid, while the performance of Primer C7 was similar to the random hexamer primer (Primer 6NS2) (Figure 1). Internal modification of the C3 spacer
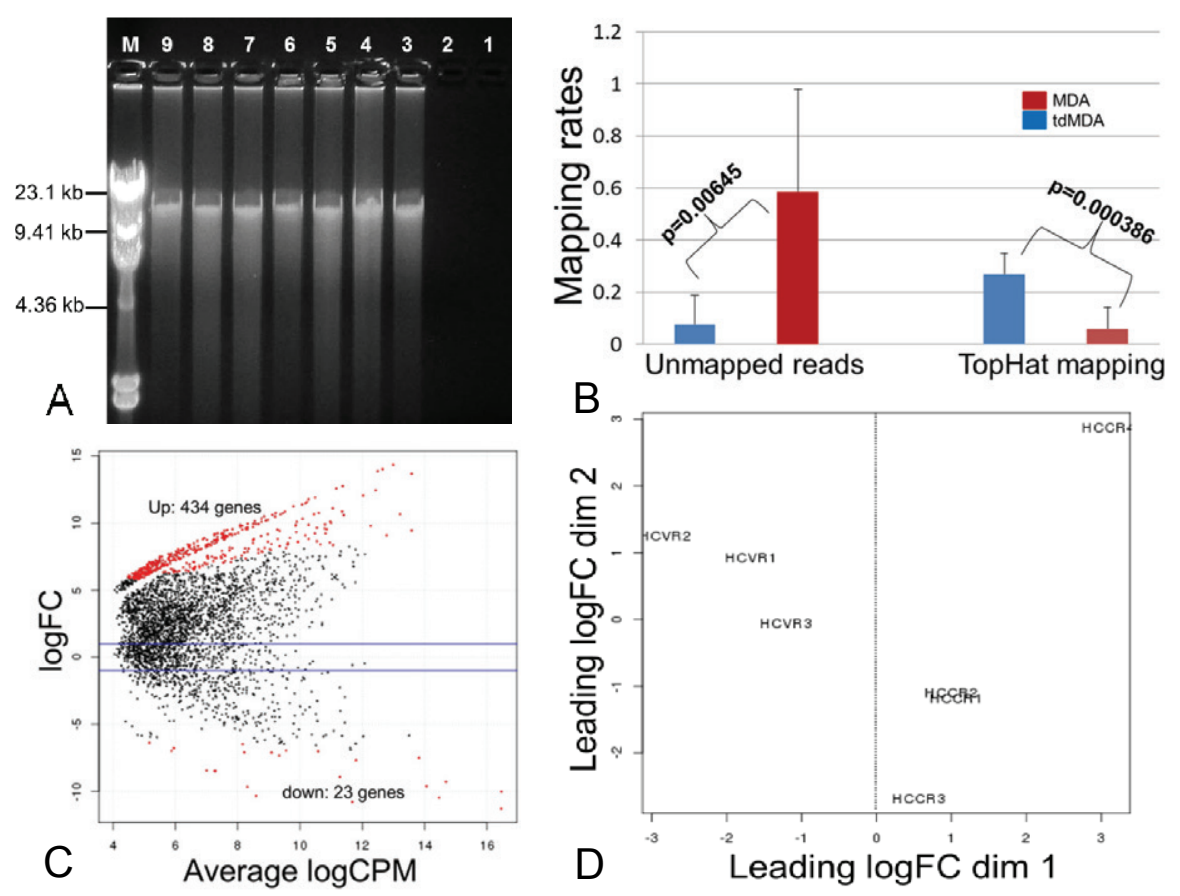

Figure 3. Transcriptome sequencing of circulating RNA. (A) RNA extracted from seven patient serum samples was amplified using real-time reverse transcription-template dependent multiple displacement amplification (RT-tdMDA). Lanes 1-2: negative control (water); Lanes 3-9: seven hepatitis $C$ virus (HCV) and hepatocellular carcinoma (HCC) patient serum samples; M: lambda DNA/HindIII marker. (B) Comparisons of the percentages of unmapped reads and TopHat mapping rates between tdMDA and regular MDA. (C) Differential gene expression (DGE) analysis in edgeR identified 457 genes showing $\geq 2$ fold changes either up- $(n=424)$ or down-regulation $(n=23)$ in the HCC group. (D) Unsupervised clustering analysis among HCV and HCC patients based on the leading log-fold-change of detected gene transcripts.

caused steric hindrance. However, such hindrance, as shown with the Group 2 primers, cannot differentiate between self and primer-template priming, which was not improved by the inclusion of LNA bases (Group 3). Despite the theoretical possibility (30), RNA primers (Group 4) were incapable of inhibiting TIA. The constrained primers in Group 5 resulted in an overall reduction of MDA yields, and TIA was still observed.

Substantial suppression of TIA was found in MDA using 5' end-blocked random hexamer (Group 6) or pentamer (Group 7) primers (Figure 1). Of the eight options to block $5^{\prime}$ ends of random pentamer primers, consecutive dSpacer (1',2'-dideoxyribose) (Primer C22), amino modifier C6 (Primer C30) and hexynyl (Primer C31) produced no amplification at all. While other options showed a substantial suppression of TIA, consistent suppression of TIA resulting in increased reproducibility was only achieved using the C18 spacer modification (Primer C28), suggesting the subtle influence of the different blocking options (Figure 1). Given the decreased melting temperatures of random pentamer primers in comparison to random hexamer primers, the incubation temperature in MDA with the Group 7 primers was initially reduced from $30^{\circ} \mathrm{C}$ to $28^{\circ} \mathrm{C}$. In subsequent experiments with Primer C28, this temperature appeared to be optimal with regard to the complete elimination of TIA and provided MDA yields comparable to the use of the random hexamer primers with or without C18 spacer-blocked 5' ends (Figure S3).

The tdMDA reaction could tolerate primer concentrations up to $400 \mu \mathrm{M}$ (Supplementary Figure S4). Beyond this threshold, yield was reduced dramatically, perhaps due to decreased $\mathrm{Mg}^{2+}$ concentration caused by binding to primer (31). For HCV plasmid amounts ranging from $1 \mathrm{ng}$ to $50 \mathrm{pg}$, tdMDA yields were maximized with primer concentrations of 80-100 $\mu \mathrm{M}$. When the input amount was lower than 50 pg in a $40-\mu \mathrm{L}$ reaction, tdMDA required templates to 
be freshly prepared. After being resuspended in water, Primer C28 with the C18 spacer modification was considerably stable. No apparent reduction in MDA yields was seen when using Primer C28 subjected to 30 thaw-freeze cycles (Supplementary Figure S5). Similarly, tdMDA was reproducible when using Primer C28 stored up to 6 months at $-20^{\circ} \mathrm{C}$ (Supplementary Figure S5).

The mechanisms for TIA generation in MDA are not fully understood. However, based on the observations from the current study, we speculate there two possibilities: (i) polymerase jumping and (ii) self-priming. It has long been known that Taq DNA polymerase is capable of jumping among templates (32). We believe that this kind of activity might be enhanced by phi29 DNA polymerase due to the polymerase's inability to be used as a molecular brake in comparison to Taq DNA polymerase (Sahu, S. PhD thesis, Duke University, 2007). Polymerase jumping among random primers could form artificial templates for the generation of TIA. This process was abolished by blocking the 5 ' ends of random primers. On the other hand, self-priming is thought to be a common mechanism responsible for artifacts in PCR and MDA (12). Our data showed no product was generated in MDA with Primer C5, which was actually 4-bp in length after digestion owing to the exonuclease activity of phi29 DNA polymerase. Thus, efficient polymerization by phi29 DNA polymerase may require 5-bp priming, which would potentially allow self-priming among random hexamer (6 bp) primers. This may explain why TIA cannot be eliminated entirely through the use of random hexamer primers with 5' ends blocked by the C18 spacer (Figure 1). Primers C1 and C2 have an internal modification of the C3 spacer that theoretically prevents self-priming. Primer C25 is a random pentamer primer without modification. The use of these primers

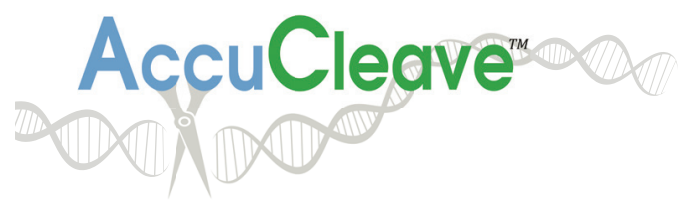

\section{Detecting CRISPR gene editing events just got QUICKR}

Introducing AccuCleave for use with agarose gels

- Simple and accurate enzymatic digestion assay

- Reduce sequencing costs and times with efficient screening methods

- Optimized Taq eliminates time consuming PCR cleanup

Streamline your genetic editing workflows

at www.aati-us.com/AccuCleave

produces a significant amount of TIA (Figure 1). Based on these observations, polymerase jumping, rather than selfpriming, might be the critical factor for TIA formation in MDA.

Finally, tdMDA was evaluated for profiling circulating RNA. The average RNA recovery from $200 \mu \mathrm{L}$ serum was $10.76 \pm 2.8 \mathrm{ng}$ using the QIAGEN miRNeasy Serum/Plasma kit. After optimizing genomic DNA removal (Supplementary Table S1), 3 serum samples with various RNA yields were selected for real-time RT-MDA with a 24-h incubation. While DNA accumulation was not observed in the negative control (water), amplification of all 3 samples with 10 -fold serial dilutions plateaued after an approximately 18-h incubation (Figure 2). The protocol was then applied to the seven HCV and HCC patient samples (Figure 3A). Illumina sequencing of RT-tdMDA product provided an average of 1.45 million reads per sample. Unmapped reads were significantly lower in tdMDA than regular MDA with random hexamer primers $(7 \pm 10.9 \%$ versus $58.6 \pm 39 \%$; $P$ $=0.006$ ) (Figure 3B). Data generated via tdMDA showed a much higher mapping rate than regular MDA in TopHat $26.9 \pm$ $7.9 \%$ versus $5.8 \pm 8.2 \% ; P=3.8 \times 10^{-4}$ ) (Figure 3B). The TopHat-feature CountsedgeR analysis detected 3459 genes in human circulating RNA. Of these 3459 genes, 457 showed $\geq 2$ fold changes, either up- ( $n=424)$ or down-regulated $(n=23)$ in the HCC group (Figure 2C). Notably, unsupervised clustering analysis showed a clear separation of HCV from HCC based on the leading log-fold-change, which is the rootmean-square average of the largest log2-fold-changes between each pair of samples (Figure 2D) (24).

Here, we reported a simple but reliable solution for the elimination of TIA in MDA. Template-dependent amplification should extend MDA's application to biological specimens with low or ultralow amounts of genetic material. By applying tdMDA, we demonstrated that efficient profiling of circulating RNA can be achieved from as little as $0.2 \mathrm{~mL}$ patient serum. Substantial differences in transcriptome profiles between HCV and HCC patients further demonstrate the promise of tdMDA for RNA-based liquid biopsy applications. 


\section{Author contributions}

X.F. and A.M.D. conceived and designed the experiments. W.W., Y.R., Y.L., and Y.X. performed the experiments. S.G., A.M.D., and X.F. interpreted the data. X.F. analyzed the data and wrote the paper.

\section{Acknowledgments}

This work was supported by the US National Institutes of Health (NIH) grants DK080711 (X.F.), Al111047 (X.F.), and a seed grant from the Saint Louis University Liver Center (X.F.). This paper is subject to the NIH Public Access Policy.

\section{Competing interests}

The authors declare no competing interests.

\section{References}

1. Nelson, J.R. 2014. Random-primed, Phi29 DNA polymerase-based whole genome amplification. Curr Protoc Mol Biol. 105:unit 15.13.

2. Dean, F.B., S. Hosono, L. Fang, X. Wu, A.F. Faruqi, P. Bray-Ward, Z. Sun, Q. Zong, et al. 2002. Comprehensive human genome amplification using multiple displacement amplification. Proc. Natl. Acad. Sci. USA 99:5261-5266.

3. Hou, Y., L. Song, P. Zhu, B. Zhang, Y. Tao, X. Xu, F. Li, K. Wu, et al. 2012. Singlecell exome sequencing and monoclonal evolution of a JAK2-negative myeloproliferative neoplasm. Cell 148:873-885.

4. Young, N.D., A.R. Jex, B. Li, S. Liu, L. Yang, Z. Xiong, Y. Li, C. Cantacessi, et al. 2012. Whole-genome sequence of Schistosoma haematobium. Nat. Genet. 44:221-225.

5. Binga, E.K., R.S. Lasken, and J.D. Neufeld. 2008. Something from (almost) nothing: the impact of multiple displacement amplification on microbial ecology. ISME J. 2:233-241.

6. Wang, W., X. Zhang, Y. Xu, A.M. Di Bisceglie, and X. Fan. 2013. Viral categorization and discovery in human circulation by transcriptome sequencing. Biochem. Biophys. Res. Commun. 436:525-529.

7. Pan, X., R.E. Durrett, H. Zhu, Y. Tanaka, Y. Li, X. Zi, S.L. Marjani, G. Euskirchen, et al. 2013. Two methods for full-length RNA sequencing for low quantities of cells and single cells. Proc. Natl. Acad. Sci. USA 110:594-599.

8. Woyke, T., A. Sczyrba, J. Lee, C. Rinke, D. Tighe, S. Clingenpeel, R. Malmstrom, R. Stepanauskas, and J.F. Cheng. 2011. Decontamination of MDA reagents for single cell whole genome amplification. PLoS One 6:e26161.

9. Wang, G., E. Maher, C. Brennan, L. Chin, C. Leo, M. Kaur, P. Zhu, M. Rook, et al. 2004.
DNA amplification method tolerant to sample degradation. Genome Res. 14:2357-2366.

10. Pan, X., A.E. Urban, D. Palejev, V. Schulz, F. Grubert, Y. Hu, M. Snyder, and S.M. Weissman. 2008. A procedure for highly specific, sensitive, and unbiased wholegenome amplification. Proc. Natl. Acad. Sci. USA 105:15499-15504.

11. Wu, L., X. Liu, C.W. Schadt, and J. Zhou. 2006. Microarray-based analysis of subnanogram quantities of microbial community DNAs by using whole-community genome amplification. Appl. Environ. Microbiol. 72:4931-4941.

12. Brukner, I., B. Paquin, M. Belouchi, D. Labuda, and M. Krajinovic. 2005. Selfpriming arrest by modified random oligonucleotides facilitates the quality control of whole genome amplification. Anal. Biochem. 339:345-347.

13. Takahashi, H., K. Yamamoto, T. Ohtani, and S. Sugiyama. 2009. Cell-free cloning using multiply-primed rolling circle amplification with modified RNA primers. Biotechniques 47:609-615.

14. Kurn, N., P. Chen, J.D. Heath, A. Kopf-Sill, K.M. Stephens, and S. Wang. 2005. Novel isothermal, linear nucleic acid amplification systems for highly multiplexed applications. Clin. Chem. 51:1973-1981.

15. Alsmadi, O., F. Alkayal, D. Monies, and B.F. Meyer. 2009. Specific and complete human genome amplification with improved yield achieved by phi29 DNA polymerase and a novel primer at elevated temperature. BMC Res. Notes 2:48.

16. Hutchison, C.A., H.O. Smith, C. Pfannkoch, and J.C. Venter. 2005. Cell-free cloning using phi29 DNA polymerase. Proc. Natl. Acad. Sci. USA 102:17332-17336.

17. Rykova, E.Y., W. Wunsche, O.E. Brizgunova, T.E. Skvortsova, S.N. Tamkovich, I.S. Senin, P.P. Laktionov, G. Sczakiel, and V.V. Vlassov. 2006. Concentrations of circulating RNA from healthy donors and cancer patients estimated by different methods. Ann. N. Y. Acad. Sci. 1075:328-333.

18. Wang, W., X. Zhang, Y. Xu, G.M. Weinstock, A.M. Di Bisceglie, and X. Fan. 2014. Highresolution quantification of hepatitis $C$ virus genome-wide mutation load and its correlation with the outcome of peginterferonalpha2a and ribavirin combination therapy. PLoS One 9:e100131.

19. Lee, W.M., J.L. Dienstag, K.L. Lindsay, A.S. Lok, H.L. Bonkovsky, M.L. Shiffman, G.T. Everson, A.M. Di Bisceglie, et al. 2004. Evolution of the HALT-C Trial: pegylated interferon as maintenance therapy for chronic hepatitis $C$ in previous interferon nonresponders. Control. Clin. Trials 25:472-492.

20. Lu, Y., Y. Xu, A.M. Di Bisceglie, and X. Fan. 2013. Comprehensive cloning of patientderived 9022-bp amplicons of hepatitis C virus. J. Virol. Methods 191:105-112.

21. Champlot, S., C. Berthelot, M. Pruvost, E.A. Bennett, T. Grange, and E.M. Geigl. 2010. An efficient multistrategy DNA decontamination procedure of PCR reagents for hypersensitive PCR applications. PLoS One 5:e13042.

22. Lareu, R.R., K.S. Harve, and M. Raghunath. 2007. Emulating a crowded intracellular environment in vitro dramatically improves RT-PCR performance. Biochem. Biophys. Res. Commun. 363:171-177.

23. Trapnell, C., A. Roberts, L. Goff, G. Pertea, D. Kim, and D.R. Kelley. 2012. Differential gene and transcript expression analysis of RNA-seq experiments with TopHat and Cufflinks. Nat. Protoc. 7:562-578.

24. Robinson, M.D., D.J. McCarthy, and G.K. Smyth. 2010. edgeR: a Bioconductor package for differential expression analysis of digital gene expression data. Bioinformatics 26:139-140.

25. Liao, Y., G.K. Smyth, and W. Shi. 2014. featureCounts: an efficient general purpose program for assigning sequence reads to genomic features. Bioinformatics 30:923930.

26. Yilmaz, S., M. Allgaier, and P. Hugenholtz. 2010. Multiple displacement amplification compromises quantitative analysis of metagenomes. Nat. Methods 7:943-944.

27. Motley, S.T., J.M. Picuri, C.D. Crowder, J.J. Minich, S.A. Hofstadler, and M.W. Eshoo. 2014. Improved multiple displacement amplification (iMDA) and ultraclean reagents. BMC Genomics 15:443.

28. Takahashi, H., H. Yamazaki, S. Akanuma, H. Kanahara, T. Saito, T. Chimuro, T. Kobayashi, T. Ohtani, et al. 2014. Preparation of Phi29 DNA polymerase free of amplifiable DNA using ethidium monoazide, an ultraviolet-free light-emitting diode lamp and trehalose. PLoS One 9:e82624.

29. Garmendia, C., A. Bernad, J.A. Esteban, L. Blanco, and M. Salas. 1992. The bacteriophage phi 29 DNA polymerase, a proofreading enzyme. J. Biol. Chem. 267:2594-2599.

30. Lagunavicius, A., E. Merkiene, Z. Kiveryte, A. Savaneviciute, V. Zimbaite-Ruskuliene, T. Radzvilavicius, and A. Janulaitis. 2009 Novel application of Phi29 DNA polymerase: RNA detection and analysis in vitro and in situ by target RNA-primed RCA. RNA 15:765-771.

31. Kramer, M.F. and D.M. Coen. 2006. Enzymatic amplification of DNA by PCR: standard procedures and optimization. Curr Protoc Cytom. Appendix 3:Appendix3K.

32. Pääbo, S., D.M. Irwin, and A.C. Wilson. 1990. DNA damage promotes jumping between templates during enzymatic amplification. J. Biol. Chem. 265:4718-4721.

Received 01 March 2017; accepted 25 May 2017.

Address correspondence to Xiaofeng Fan or Adrian M, Di Bisceglie, Division of Gastroenterology \& Hepatology, Department of Internal Medicine, Saint Louis University School of Medicine, St. Louis, MO 63104. E-mail: fanx@slu.edu (X.F.) or dibiscam@slu.edu (A.M.D.)

To purchase reprints of this article, contact: biotechniques@fosterprinting.com 